\title{
(Alguns) desafios para os Produtos Educacionais nos Mestrados Profissionais nas áreas de Ensino e Educação
}

\section{(Some) challenges on Educational Products Elaboration in Professional Master Courses in Teaching and Education areas}

\author{
Carmen Érica Lima de Campos Gonçalves \\ Instituição Federal de Educação, Ciência e Tecnologia do Amazonas \\ carmen.erica@hotmail.com \\ Carolina de Souza Oliveira \\ Instituição Federal de Educação, Ciência e Tecnologia do Amazonas \\ acarolliveira@gmail.com \\ Gilmara Oliveira Maquiné \\ Instituição Federal de Educação, Ciência e Tecnologia do Amazonas \\ gilmaramaquine@gmail.com \\ Andréa Pereira Mendonça \\ Instituição Federal de Educação, Ciência e Tecnologia do Amazonas \\ andrea.mendonca@ifam.edu.br
}

\section{Resumo}

A elaboração de produtos é uma obrigatoriedade nos Mestrados Profissionais e, em particular, nos cursos da área de Ensino e Educação, é imperativo que as pesquisas realizadas resultem em um produto educacional com potencial de inserção na Educação Básica, Profissional e Ensino Superior. Para que esta inserção aconteça, é necessário superar alguns desafios (linguagem, capacidade de replicação, internacionalização, disponibilidade e acessibilidade) a fim de maximizar as oportunidades de alcance e uso dos produtos educacionais pelo público a que se destina. Neste artigo, foi conduzida uma investigação qualitativa, segundo uma estratégia exploratória sequencial, em duas fases: uma fase inicial de coleta quantitativa de dados (catalogação de produtos educacionais), seguida de uma fase de análise qualitativa dos dados coletados, segundo critérios estabelecidos na literatura e legislação sobre produtos educacionais. A investigação resultou em um conjunto de produtos educacionais, consolidados no cenário nacional e internacional, que apontam reflexões e possíveis caminhos para subsidiar o trabalho de professores, estudantes e gestores de mestrados profissionais para a ampliação do estudo 
ISSN: 2446-774X

sobre produtos educacionais, assim como o enfrentamento dos desafios para sua elaboração e inserção social.

Palavras-chave: Produto educacional. Mestrado profissional. Ensino tecnológico.

\begin{abstract}
The elaboration of products is a mandatory requirement in Professional Master Courses. In Education and Teaching Areas, in particular, it is important that these resulting educational products have the potential to be adopted into basic, professional and higher education activities. To endorse this adoption, some challenges need to be overthrown when elaborating such products to efficiently reach the targeted audience, such as: language, replicability, internationalization, availability and accessibility. Considering this perspective, in this paper we carried out a qualitative investigation as an exploratory sequential strategy of two phases: a quantitative collection of educational products followed by their qualitative analysis, respecting criteria suggested both in literature and in legislation of educational products. As a result, we present the derived analysis of both national and international educational products, also pointing out some guidelines of possible strategies to overcome the previously mentioned challenges. The results can support the elaboration of new educational products, the adaptation of existing materials and also encourage other studies on educational products in face of the challenges on their elaboration.
\end{abstract}

Key words: Educational product. Technological teaching. Professional master course.

\title{
Introdução
}

Os Mestrados Profissionais (doravante MP) têm como um de seus objetivos capacitar profissionais qualificados para o exercício da prática profissional avançada e transformadora de procedimentos, visando atender demandas sociais, organizacionais ou profissionais e do mercado de trabalho (BRASIL, 2009).

O interesse por MP teve seu início datado da década de 90, porém somente em 1998 foi publicada a Portaria oㅡ 80, pela Coordenação de Aperfeiçoamento de Pessoal de Nível Superior (CAPES), a qual reconhece os MP (BARBOSA, 2016).

Até 2016 o portal da CAPES registrava o total de 574 MP no Brasil, sendo 63 da área de Ensino e 38 da área de Educação (BARBOSA, 2016). Atualmente, constam 767 MP dos quais 81 são da área de Ensino e 46 são da área de Educação (CAPES, 2018). Esse deslanche se deu após a publicação da normativa de 2009 que trouxe a compreensão de que os MP devem atender o mundo do trabalho e o sistema produtivo devolvendo profissionais qualificados, cujo perfil de egresso deve deter a intervenção geradora de produtos que 


\section{aEducitec \\ ISSN: $2446-774 X$}

melhorem o ambiente de trabalho de onde esse egresso é oriundo ou está inserido.

A CAPES desde 1976 é quem do Ministério da Educação - MEC desenvolve atividades direcionadas à pós-graduação stricto sensu no Brasil, gerenciando, avaliando e mediando os cursos de forma a regulá-los e mantê-los no padrão de qualidade desejado, o que fomenta as políticas para esta área e os investimentos a ela direcionados (CAPES, 2017), o que inclui as diretrizes sobre o Mestrado Profissional e os produtos dele derivados. Duas são as portarias de onde parte toda a regulamentação sobre os cursos de pós-graduação na modalidade profissional (CAPES, 2014, 2018): a Portaria (CAPES) n. 131/2017 e a portaria MEC n. 389/2017.

Em 2017, os MP no Brasil foram agrupados dentro de vinte áreas, entre as quais estão a Educação e o Ensino (como áreas distintas) e a CAPES (2017) destaca que a Portaria Normativa no17/2009 atualizada pela Portaria no131/2017 oportunizou o crescimento desta modalidade de pós-graduação; porém a identidade destes cursos está diretamente relacionada ao tempo de existência deles, o que evidencia a imperativa necessidade de discutir para avançar a temática, vinculando diretamente também ao estabelecimento do que seja um de seus diferenciais: o produto derivado da pesquisa que construiu a dissertação.

Neste sentido, os Programas de Pós-Graduação (PPG) têm buscado maneiras de aperfeiçoar o registro, avaliação e valorização das atividades de desenvolvimento de produtos, buscando ampliar a inserção destes nos ambientes organizacionais e profissionais. No caso dos programas de PósGraduação na área de Ensino e Educação, o foco está voltado para o desenvolvimento de produtos educacionais, primando por sua inserção no Sistema Educacional Brasileiro (CAPES, 2016b).

Cabe destacar, porém, que o desenvolvimento de produtos não é uma atividade trivial e revela vários desafios, dentre eles: a adoção de linguagem apropriada ao público a que se destina; capacidade de replicação por terceiros; internacionalização; disponibilidade para livre acesso e acessibilidade por pessoas com deficiência.

A fim de contribuir para o melhor entendimento destes desafios e dos possíveis caminhos para a sua superação pelos PPG na área de Educação e Ensino, conduzimos uma investigação qualitativa exploratória sequencial, em duas fases, adaptada de Creswell (2014): uma fase inicial de coleta quantitativa de dados, no caso desta pesquisa, de coleta de produtos educacionais, seguida de uma fase de análise qualitativa dos produtos coletados, segundo critérios estabelecidos na literatura e legislação sobre produtos educacionais.

De acordo com Creswell (2014), esta estratégia prioriza o aspecto qualitativo do estudo. Assim, no nível mais básico, o objetivo desta estratégia é usar dados e resultados quantitativos para auxiliar na interpretação e resultados qualitativos. Seu método em duas fases o torna mais fácil de implementar e direto para descrever e relatar, sendo útil para o pesquisador que quer explorar um fenômeno, mas que também quer expandir os resultados qualitativos. 


\section{Eteducitec}

ISSN: $2446-774 X$

No caso desta pesquisa, o objetivo da aplicação desta metodologia foi o de viabilizar a identificação de produtos educacionais consolidados nacional e internacionalmente e compreender como as características destes produtos podem ser consideradas na concepção e elaboração dos produtos educacionais derivados das pesquisas desenvolvidas nos Mestrados Profissionais.

Uma pesquisa desta natureza é relevante porque, embora um dos diferenciais dos mestrados profissionais em Educação e Ensino seja a elaboração de produtos educacionais, uma problemática enfrentada pelos cursos de PósGraduação é a dificuldade de inserção social destes produtos e consequente uso na Educação Básica, Profissional e Ensino Superior. Assim, os resultados desta pesquisa podem prover indicadores que auxiliem professores, estudantes e gestores de mestrados profissionais na concepção e elaboração de produtos educacionais com maior visibilidade e alcance pelo público a que se destina.

\section{Produtos Educacionais nos Mestrados Profissionais e seus Desafios}

Pensar "Produto" submete à observação semântica que diz ser "Qualquer coisa fabricada. O que é produzido, destinado ao consumo próprio ou ao comércio. Aquilo que resulta de uma atividade humana." (MICHAELIS, 2018), vamos apresentar no decorrer do texto que aquilo que é compreendido por produto derivado de pesquisa em pós-graduação profissional não se figura muito longe da semântica, apenas amplia o entendimento quando situa na área a que se destina.

Produtos educacionais segundo a CAPES (2013), podem ser caracterizados como processos ou produtos educativos utilizados e utilizáveis em condições reais de ensino, como protótipo ou de cunho artesanal. Ressaltamos ainda que o produto em questão, fruto da pesquisa aplicada deve ser "identificável e independente da dissertação" (MOREIRA; NARDI, p. 4, 2009). Esses autores afirmam também que embora o produto seja resultado da dissertação, ele tem "identidade própria".

Processos e produtos educacionais devem ser passíveis de replicação por outros profissionais de ensino e podem ser (CAPES, 2013, p.53):

1. Mídias educacionais (vídeos, simulações, animações, experimentos virtuais, áudios, objetos de aprendizagem, aplicativos de modelagem, aplicativos de aquisição e análise de dados, ambientes de aprendizagem, páginas de internet e blogs, jogos educacionais, etc.)

2. Protótipos educacionais e materiais para atividades experimentais

3. Propostas de ensino (sugestões de experimentos e outras atividades práticas, sequências didáticas, propostas de intervenção, e etc.)

4. Material textual (manuais, guias, textos de apoio, artigos em revistas técnicas ou de divulgação, livros didáticos e paradidáticos, histórias em quadrinhos e similares)

5. Materiais interativos (jogos, kits e similares) 


\section{Ed Educitec \\ ISSN: $2446-774 X$}

6. Atividades de extensão (exposições científicas, cursos de curta duração, oficinas, ciclos de palestras, exposições, atividade de divulgação científica e outras)

7. Desenvolvimento de aplicativos

8. Organização de evento

9. Programa de rádio e TV

10. Relatórios de pesquisa

11. Patentes (depósito, concessão, cessão e comercialização)

12. Serviços técnicos.

A partir da categorização acima mencionada, a CAPES (2016a) destaca quatro parâmetros para classificação dos produtos:

(1) Validação Obrigatória do produto por comitês ad hoc, órgão de fomento ou banca de dissertação, (2) Registro do Produto, que expressa sua vinculação a um sistema de informações em âmbito nacional ou internacional, como por exemplo, ISBN, ISSN, ANCINE, Registro de Domínio, Certificado de Registro Autoral, Registro ou Averbação na Biblioteca Nacional, além de registros de patentes e marcas submetidos ao INPI, (3) Utilização nos sistemas de educação, saúde, cultura ou CT\&I, que expressa o demandante ou o público alvo dos produtos, e (4) Acesso livre (online) em redes fechadas ou abertas, nacionais ou internacionais, especialmente em repositórios vinculados a Instituições Nacionais, Internacionais, Universidades, ou domínios do governo na esfera local, regional, ou federal [...] (CAPES, p. 16, 2016).

Esses parâmetros pautam a validação e classificação dos produtos educacionais resultantes dos MP. Nessa validação devem ainda os produtos tecnológicos educacionais e sociais conter Sinopse de até 60 palavras descrevendo o produto, público alvo, URL do produto e até 3 imagens do produto em arquivos anexos (CAPES, 2013).

Os materiais, dissertações e seus produtos, são armazenados no repositório da CAPES, chamado EDUCAPES (http://educapes.capes.gov.br), cujo acesso é livre e gratuito para qualquer pessoa interessada, ainda que o público-alvo sejam professores e alunos de qualquer nível educacional.

O EDUCAPES foi criado através do software DSpace (http://www.dspace.org), o mesmo utilizado por Instituições de todo o mundo que ensejam divulgar seus materiais e tê-los de forma acessível ao público em geral. Este formato permite inclusive que o usuário receba alerta sobre novos conteúdos.

Para figurar no repositório EDUCAPES, a autoria deve ser oriunda de alguma Universidade do programa Universidade Aberta do Brasil (UAB), com opção de licença e sujeito à moderação pelos usuários do portal.

Conforme descrevemos anteriormente, há uma série de desafios para a elaboração de produtos educacionais nos MP, e neste trabalho destacamos cinco (05) deles, conforme explicitados a seguir:

1. Linguagem: capacidade de expressar o conteúdo do produto educacional de forma clara, correta e adequada; que faz relação direta com sua apresentação provendo a qualidade visual e organizacional a fim de tornálo mais intuitivo e receptivo para o público alvo a que se destina; 


\section{Ceducitec \\ ISSN: $2446-774 X$}

2. Capacidade de replicação: potencial do produto de ser reproduzido e/ou utilizado pelo público alvo a que se destina;

3. Internacionalização: possibilidade de replicação e/ou utilização do produto por público alvo de outros países cujo idioma é diferente daquele adotado pelo país de origem do produto;

4. Disponibilidade: tornar o produto disponível em redes, repositórios e/ou plataformas que permitam o alcance pelo público alvo; possibilitando a divulgação e popularização

5. Acessibilidade: qualidade de tornar acessível o produto educacional por pessoas com deficiência, de forma autônoma e segura, totalmente ou parcialmente assistido.

Tendo em vista estes desafios, apresentamos na próxima seção o percurso metodológico adotado para, a partir da identificação de produtos educacionais consolidados nacional e internacionalmente, identificar possíveis caminhos que possam subsidiar o trabalho de professores, estudantes e gestores de mestrados profissionais para a ampliação do estudo sobre produtos educacionais e o enfrentamento dos desafios para sua elaboração.

\section{Percurso Metodológico para Identificação e Análise de Produtos Educacionais}

Conforme descrevemos anteriormente, o percurso metodológico de nossa pesquisa seguiu uma abordagem qualitativa exploratória sequencial, em duas fases (Creswell, 2014): a primeira, de coleta quantitativa de dados, e a segunda de análise qualitativa dos dados. Cabe ressaltar, que no caso desta pesquisa, os dados se referem a produtos educacionais.

\section{Primeira fase: coleta quantitativa de dados}

Inicialmente, realizamos uma pesquisa exploratória em plataformas digitais (por exemplo, EduCapes e Repositórios Institucionais de Universidades e Institutos Federais) máquina de buscas (Google e Google Scholar) e sites especializados (porvir.org, lojas virtuais de aplicativos, etc.) a fim de identificar produtos educacionais, originários de universidades ou não, que tivessem como foco formação de professores ou recursos para sala de aula. Como resultados desta atividade, obtivemos uma amostra de 40 (quarenta) produtos educacionais.

Estes produtos foram catalogados em uma planilha do Excel, contendo os seguintes campos: nome do produto, endereço eletrônico (url), status do produto (se permitia replicação ou não), categoria (obedecendo classificação da CAPES - mídias educacionais, protótipos educacionais, propostas educacionais, etc.) e um campo de comentário, no qual era realizado uma descrição sucinta do produto com comentário qualitativo sobre o mesmo. Após esse processo de catalogação, foi iniciada a segunda fase, conforme descrito a seguir.

\section{Segunda fase: análise qualitativa dos dados}


ISSN: $2446-774 X$

Com base na amostra coletada na primeira fase, realizamos uma análise qualitativa dos produtos pautada nos cinco critérios (desafios) descritos anteriormente: linguagem, capacidade de replicação, internacionalização, disponibilidade e acessibilidade.

Nesta fase, cada produto da amostra foi analisado a fim de verificar aqueles que melhor demonstrassem evidências de superação dos cinco (05) desafios mencionados anteriormente.

Neste processo de análise, os produtos foram testados/experimentados a fim de permitir melhor conhecimento de suas características e limitações, sempre tomando como referência os cinco critérios mencionados. Foi realizada uma investigação sobre os autores, o histórico de cada produto, sua reputação (avaliação) pelos usuários, assim como os mecanismos utilizados para sua divulgação.

Esta análise resultou na seleção de sete (07) produtos educacionais, conforme apresentamos no Quadro 1.

Quadro 1 - Seleção e descrição dos produtos educacionais (continua)

FORMAÇÃO DE PROFESSORES

PRODUTO: Livro Aula Nota 10 (LEMOV, 2011)

O livro foi publicado em 2010 nos Estados Unidos com o título Teach Like a Champion, tendo mais de 150 mil cópias vendidas. O autor, Doug Lemov é Diretor Executivo da organização não governamental Uncommon Schools, cujo trabalho é supervisionar redes de escolas em Nova York; e sua compilação foi feita a partir da observação da atuação de professores que apresentavam resultados positivos em avaliações externas mesmo vivenciando situações adversas no cotidiano das salas de aula.

Endereço eletrônico: https://goo.gl/MEZQdX

PRODUTO: Livro Desafios reais do cotidiano escolar brasileiro - 22 dilemas vividos por diretores, coordenadores e professores em escolas de todo o Brasil (INSTITUTO PENÍNSULA, 2018)

PRODUTO: Livro Desafios reais do cotidiano escolar brasileiro (cont.)

O livro apresenta 22 dilemas vividos por diretores, coordenadores e professores em escolas de todo Brasil. Inspirado em um programa liderado pelo David Rockefeller Center for Latin American Studies (DRCLAS), da Universidade de Harvard, para desenvolver e publicar casos escritos por diretores de escolas no Chile, o projeto tem como objetivo ampliar o conhecimento da metodologia de desenvolvimento de casos para um novo público: os professores do Brasil. Endereço eletrônico: https://goo.gl/FrKmQU

PRODUTO: InnoveEdu

Portal de experiências que disponibiliza a compilação de 96 casos práticos de inovações em educação identificados em todo o mundo. Foi realizada pelo Porvir, em parceria com três organizações que possuem experiência em pesquisa e disseminação de inovações educacionais de diferentes países: Edsurge (Estados Unidos), Innovation Unit (Reino Unido) e WISE (Catar).

Endereço eletrônico: https://goo.gl/X2ox2f 


\section{Educitec}

ISSN: $2446-774 X$

Quadro 1 - Seleção e descrição dos produtos educacionais (conclusão)

RECURSOS PARA ADOTAR NAS SALAS DE AULA

PRODUTO: Google Expeditions

É um dos produtos Google voltada ao ensino com realidade virtual, em formato de aplicativo. Proporciona excursões virtuais em qualquer lugar, fazendo uso do Google Cardboard. Em seu repositório, possui mais de 500 expedições (passeios virtuais) prontas para uso e muitas outras estão sendo criadas colaborativamente. $O$ ambiente é composto de coleções de imagens em $3 \mathrm{D}$ e de 360 graus e, ao mesmo tempo, destaca os locais interessantes que surgem pelo caminho. O principal objetivo da ferramenta é viabilizar o ensino por meio de expedições virtuais com realidade virtual, onde o professor assume o papel de 'guia' e os alunos 'exploradores'.

Endereço eletrônico: https://goo.gl/y73Udz

PRODUTO: Primo

O primo é um jogo composto por: Robô Cubetto, o tabuleiro de programação, blocos de instruções, que funcionam em conjunto. Foi criado pela PrimoToys, empresa fundada pelo brasileiro Filippo Yacob e Matteo Loglio. A ideia inicial surgiu durante o Mestrado em Estudos Avançados de Design, realizado por Matteo Loglio na Suíça. Depois disto, os dois juntos, inspirados no trabalho de Seymour Papert cujo trabalho resultou na Linguagem LOGO. Eles consideram que a habilidade de programar computadores será essencial daqui a alguns anos, e que as pessoas deverão não só consumir tecnologia mas também criá-la.

Endereço eletrônico: https://goo.gl/6VZvuB

\section{PRODUTO: Palma Kids}

Jogo educativo que auxilia no processo de alfabetização de crianças a partir do 4 anos de idade. Foi criado pela empresa brasileira les2. A empresa les2 foi fundada pelo Professor José Luís Poli, com o objetivo de desenvolver tecnologia a serviço da educação. Além do PalmaKids a empresa tem outros aplicativos voltados para educação.

Endereço eletrônico: https://goo.gl/N9618f

PRODUTO: Star Walk 2 : Astronomia em Português para Crianças

$\mathrm{O}$ aplicativo foi criado para o ensino de astronomia com o objetivo de proporcionar a experiência de observação de estrelas. Foi criado pela empresa Vito Technology, empresa americana fundada por Victor Toporkov com especialidade em desenvolvimento, design e personalização para iOS e Android.

Endereço eletrônico: https://goo.gl/cZH2Tj

Fonte: Próprias autoras (2018).

Os resultados desta segunda fase da análise são apresentados na próxima seção.

\section{Resultados da Análise dos Produtos Educacionais}

Para a apresentação dos resultados, destacamos para cada desafio, a análise realizada e possíveis caminhos para a sua superação pelos mestrados profissionais.

\section{Linguagem}

De maneira geral, observamos que os produtos apresentados possuem uma linguagem voltada para o público-alvo a que se destina, e expressam de forma clara o seu conteúdo, bem como há preocupação com sua apresentação, de modo a tornar a utilização e manuseio do produto fácil e intuitivo.

Como exemplos, citamos o Livro 22 Desafios e o aplicativo Palma Kids. O livro apresenta casos de ensino como material didático para formação de professores numa linguagem de fácil compreensão e assimilação, causando um efeito de 


\section{arducitec \\ ISSN: $2446-774 X$}

empatia nos leitores. Por ter como público professores, gestores $\mathrm{e}$ coordenadores educacionais, a apresentação do livro é dividida em seções as quais tratam de temas pertinentes a prática desse público. No aplicativo Palma Kids a linguagem é voltada para crianças e por este motivo, toda a experiência de utilização foi criada pensando nesse público. A interface do aplicativo que tem como objetivo auxiliar no ensino de alfabetização, apresenta-se de maneira lúdica e intuitiva, explorando cores, áudio, vídeo e conteúdo para aproximar-se do público alvo.

Ao analisarmos o conjunto de produto demonstrado no Quadro 1, observamos que a linguagem é um aspecto importante para promoção da inserção do produto junto ao público a que se destina. Desta forma, o desafio para os estudantes dos mestrados profissionais está em conceber produtos com uma linguagem diferente daquela adotada na escrita da dissertação. Isto por sua vez, demanda um processo de elaboração específica, não sendo possível simplesmente (re)aproveitar textos da dissertação para o produto. A linguagem, neste caso, é expressa não apenas pelo texto em si, mas pelo conjunto dos elementos que compõem a sua apresentação: figuras, infográficos, quadros e os demais recursos estéticos que facilitam a identificação das informações e tornam a leitura mais intuitiva e agradável. Considerando que o aspecto visual do produto tem impacto na adesão pelo público alvo, é importante que os estudantes de mestrado considerem a possibilidade de consultar ou ter assessoria de outros profissionais, tais como designers, para a elaboração de seus produtos educacionais. Os produtos analisados dão evidências de que outros profissionais trabalharam em conjunto com os autores para a superação deste desafio.

\section{Capacidade de replicação}

De maneira geral, observamos que os produtos analisados são altamente replicáveis, respondendo ao desafio de utilização e reprodução pelo público alvo.

Como exemplos citamos o livro Aula nota 10 (LEMOV, 2011) e o aplicativo Google Expedições. O livro Aula Nota 10 reúne 49 técnicas que podem facilmente ser utilizadas e replicadas nas salas de aula, nele estão contidas todas as orientações para que os professores reproduzam a aplicação das técnicas. O Google Expedições, por sua vez, é um aplicativo que pode ser utilizado em aulas de diferentes disciplinas, e evidencia a capacidade de replicação pelo guia de utilização, no qual apresenta todos os requisitos para realização de uma expedição virtual utilizando o Google Expedições.

Ao analisarmos o conjunto de produtos descritos no Quadro 1, observamos que a capacidade de replicação é um aspecto de suma importância para a efetiva utilização dos produtos pelo público a que se destina. Desta forma, o desafio para os estudantes de mestrados profissionais está na necessidade de pensar como o produto será replicado fazendo uso de materiais facilitadores tais como: manuais, guias, vídeos instrucionais e outros materiais de apoio. Ao facilitar o processo de replicação, naturalmente ampliamos as possibilidades de validação do produto pela comunidade. 


\section{aEducitec}

ISSN: $2446-774 X$

$\overrightarrow{\mathrm{E}}$ importante destacarmos que capacidade de replicação e linguagem, mencionado anteriormente, estão diretamente relacionados, na medida que a adoção de linguagem e design apropriados ampliam as possibilidades de replicação.

\section{Internacionalização}

Dos produtos analisados, somente alguns atendem a este desafio, considerando a perspectiva de adoção de diferentes idiomas para o mesmo produto. $O$ InnoveEdu, por exemplo, é uma plataforma que oferece o conteúdo nos idiomas português e inglês. O mesmo ocorre com o aplicativo Palma Kids, que possibilita a realização de atividades com a adoção do idioma inglês e português. Em cada idioma escolhido, os vídeos e áudios são traduzidos automaticamente, nos quais os narradores são crianças.

Ao analisarmos o conjunto de produtos descritos no Quadro 1, observamos que a internacionalização é uma característica que proporciona um diferencial aos produtos. O fato do produto estar disponível em mais de um idioma facilita a utilização deste em diferentes países. Desta forma, o desafio para os estudantes de mestrados profissionais está em viabilizar a tradução para outro idioma, além do português. Neste sentido, acreditamos que o estabelecimento de parcerias, por exemplo, entre os Programas de Pós-Graduação e os Centros de Idiomas ou Cursos de Línguas, podem facilitar a superação deste desafio.

\section{Disponibilidade}

De maneira geral os produtos analisados estão disponíveis em diferentes plataformas e formatos. Identificamos também que cada produto assume uma estratégia para popularizar seu uso e alcançar seu público alvo, buscando parceiros que contribuem na efetivação do processo de divulgação.

Por exemplo, o portal InnoveEdu disponibiliza 96 experiências na web (http://innoveedu.org/sobre). Além disso, essas experiências podem ser encontradas no site do PORVIR (http://porvir.org/), e nas redes sociais por meio do Facebook e Twitter.

Quando analisamos o jogo Primo, verificamos que sua popularização se deu a partir de participação em feiras e por campanhas de financiamento coletivo (crowdfunding) de 2013 a 2018. Após a primeira campanha, muito bem sucedida, foram criadas outras três para financiar coletivamente a produção do jogo, captando mais de $\$ 2.300 .000$ advindos de 10.000 apoiadores. O aplicativo Star Walk, por sua vez, é gratuito e está disponível nas lojas de aplicativos, ganhou destaque na Play Store ficando na lista dos melhores aplicativos de 2017. Possui mais de 500.000 instalações em aparelhos Android, pode ser vista além dos prêmios nas lojas de aplicativos, nas resenhas publicadas e nos feedbacks dos usuários.

Ao analisarmos o conjunto de produtos descritos no Quadro 1, observamos que a disponibilidade é um desafio que pode ser superado a partir da utilização de diferentes estratégias para posicionar o produto. A disponibilidade está diretamente relacionada a utilização e ao alcance das pessoas de maneira geral, pois a escolha de repositórios que proporcionem visibilidade e promoção do 


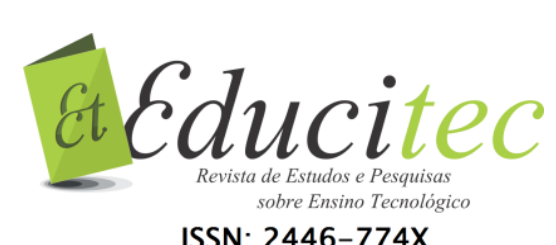

ISSN: $2446-774 X$

produto são fatores importantes para popularizá-los. Neste sentido, os produtos educacionais, além de serem disponibilizados nos sites dos cursos de PósGraduação, Repositórios Institucionais e EDUCAPES, precisam ser disponibilizados em outras mídias e plataformas apropriadas. Assim, a superação deste desafio requer identificação dos locais e meios que o público utiliza para ter acesso aos produtos.

\section{Acessibilidade}

Em nossa análise, creditamos à acessibilidade um dos maiores desafios a ser superado. Embora seja um aspecto indiscutivelmente relevante na construção dos produtos, verificamos nos produtos analisados ausência de recursos que possibilitem o seu uso por pessoas com deficiência.

Essa ausência, por sua vez, abre perspectivas no sentido de induzir o desenvolvimento de pesquisas e produtos que promovam a inclusão.

Para superar o desafio da acessibilidade é necessário considerarmos aspectos de design universal, referente ao desenvolvimento de produtos e ambientes que possam ser utilizados por todas as pessoas sem necessidades de adaptações ou design especializado (W3C, 2013), proporcionando a utilização dos produtos sem barreiras aos diferentes públicos. Além disso, é importante viabilizar cooperação entre os Programas de Pós-Graduação e os NAPNE's (Núcleo de Atendimento a Pessoas com Necessidades Especiais), escolas e associações de assistência a pessoas com deficiência a fim de criar oportunidades para a elaboração de produtos mais acessíveis.

\section{Considerações finais}

Neste artigo, apresentamos os resultados de uma investigação qualitativa que teve como foco a análise de produtos educacionais segundo cinco critérios (desafios) estabelecidos na literatura da área e legislação da CAPES: linguagem, capacidade de replicação, internacionalização, disponibilidade e acessibilidade.

O percurso metodológico obedeceu a uma estratégia exploratória sequencial, em duas fases: uma fase inicial de coleta quantitativa de dados, isto é, de catalogação de produtos educacionais (nome, endereço eletrônico, status do produto, categoria e descrição), resultando em 40 (quarenta) produtos, em nível nacional e internacional, originários de universidade ou não. A segunda fase foi destinada a uma análise qualitativa, pautada nos cinco critérios descritos anteriormente, e que teve por objetivo identificar aqueles produtos que melhor demonstrassem evidências de superação dos cinco (05) desafios mencionados anteriormente. Nesta fase, a amostra inicial composta por 40 (quarenta) produtos foi reduzida para 07 (sete), e esta redução já é um indicativo de que é um verdadeiro desafio para universidades, empresas e profissionais independentes atender integralmente aos cinco critérios para elaboração de produtos educacionais.

De acordo com a análise realizada neste trabalho, dois critérios destacam-se como os maiores desafios: acessibilidade e internacionalização. De acordo com 


\section{Eteducitec \\ ISSN: $2446-774 X$}

a pesquisa, há uma escassez de produtos que incorporem recursos de acessibilidade (como libras ou braile, por exemplo) a fim de atender públicos minoritários, sendo esta uma questão a ser melhor discutida nas PósGraduações a fim de atender a um público mais diversificado.

O segundo desafio diz respeito a internacionalização, isto é, possibilitar o acesso dos produtos em vários idiomas. Conforme observado, alguns autores preferem adotar o inglês por ser um idioma considerado universal. Contudo, no contexto educacional, a falta de familiaridade de professores e alunos com este idioma é fator restritivo para o uso. No caso de autores brasileiros, por sua vez, elaborar os produtos apenas em português, diminui significativamente as chances de inserção dos produtos em outros países. Assim, é desejável que as PósGraduações no Brasil desenvolvam parcerias com centros de idiomas a fim de possibilitar a tradução dos produtos para outros idiomas, tais como, inglês e espanhol.

No que diz respeito as contribuições deste trabalho, destacamos duas como principais: (i) subsidiar os Programas de Pós-Graduação que oferecem cursos na modalidade Profissional nas discussões sobre a elaboração de produtos no contexto acadêmico e os recursos (humanos, técnicos, legislação, etc.) necessários para este fim; (ii) ampliar a visão dos estudantes de Pós-Graduação sobre os desafios inerentes a elaboração de produtos educacionais e de alguns caminhos possíveis para superá-los.

A fim de ampliar a socialização dos resultados deste trabalho, desenvolvemos um site (https://goo.gl/8AfuvD) que detalha os produtos analisados, demonstrando de forma mais ampla suas características, assim como os resultados da análise desenvolvida. A partir do site, o leitor pode acessar os produtos e ter uma experiência pessoal que oportunize novas reflexões que podem ser retornadas às autoras de forma a amplificar a discussão sobre o tema. Também foram realizados dois colóquios para socialização e divulgação dos resultados deste trabalho para professores e estudantes de mestrados profissionais e de graduação (https://bit.ly/2CCWGEj e https://bit.ly/2KdlhBL).

Como trabalhos futuros destacamos primordialmente: (i) A importância de ampliar a análise de produtos abrangendo outros desafios (tais como, validação do produto, proteção de autoria, geração de registros e patentes); (ii) Avaliar a inserção social dos produtos educacionais advindos dos Programas de PósGraduação na modalidade profissional, a fim de subsidiar uma análise mais profunda sobre os desafios a serem enfrentados na elaboração de produtos educacionais no contexto acadêmico; e (iii) Ampliar os espaços de estudo, discussão e socialização dos conhecimentos sobre produtos educacionais em Programas de Pós-Graduação.

\section{Agradecimentos}

Agradecemos à Universidade Federal do Amazonas (UFAM) e a Fundação de Amparo à Pesquisa do Estado do Amazonas (FAPEAM) pelo apoio concedido. 


\section{Referências}

BRASIL. Edital de Convocação 25/2018 - SEB. Edital de convocação para o processo de inscrição, avaliação e precificação de tecnologias educacionais para a educação básica. Brasília: MEC, 2018.

BRASIL. Portaria n.17 de 28 de dezembro de 2009. Dispõe sobre o mestrado profissional no âmbito da Fundação Coordenação de Aperfeiçoamento de Pessoal de Nível Superior - CAPES. Diário Oficial da União, n. 248 de 29 de dezembro de 2009, seção I.

BRASIL. Portaria n.389 de 23 de março de 2017. Dispõe sobre o mestrado e o doutorado profissional no âmbito da pós-graduação stricto sensu. Brasília: MEC, 2017. Diário Oficial da União n.58 de 24 de março de 2017, p. 61. ISSN 16777042.

BRASIL. Portaria n.7 de 22 de junho de 2009. Dispõe sobre o mestrado profissional no âmbito da Fundação Coordenação de Aperfeiçoamento de Pessoal de Nível Superior - CAPES. Diário Oficial da União, n.117 de 23 de junho de 2009, p. 31, seção I.

BARBOSA, V. A gênese dos Mestrados Profissionais em Educação nas Universidades Brasileiras, Plurais Revista Multidisciplinar, Salvador, v. 1, n. 2, p. 94-113, 2016.

CAPES. Considerações sobre classificação de produção técnicaeducacional. Brasília: MEC/CAPES, 2016.

CAPES. Coordenadores de área falam sobre a avaliação de mestrados profissionais. Brasília: MEC/CAPES, 2017.

CAPES. Cursos Avaliados e Reconhecidos. Brasília: MEC/CAPES, 2018.

CAPES. Documento de área 2013. Brasília: CAPES, 2013.

CAPES. Documento de área 2016 - Ensino. Brasília: CAPES, 2016.

CAPES. FAQ. Brasília: CAPES, 2018.

CAPES. Legislação específica. Brasília: MEC/CAPES, 2014, 2018.

CAPES. Mestrado profissional. Brasília: MEC/CAPES, 2015.

CAPES. Mestrado profissional: o que é?. Brasília: MEC/CAPES, 2014, 2018.

CAPES. Plataforma Sucupira. Brasília: MEC/CAPES, 2016.

CAPES. Portaria n.131 de 28 de junho de 2017. Dispõe sobre o mestrado e o doutorado profissionais. Brasília: MEC, 2017. Diário Oficial da União n.124 de 30 de junho de 2017, p.17. ISSN 1677-7042.

CAPES. Sobre a CAPES. Brasília: MEC/CAPES, 2017.

CAPES. UFPA reúne coordenadores de mestrados profissionais de todo o Brasil. Brasília: MEC/CAPES, 2011, 2014.

CRESWELL, John W. Research Design: Qualitative, Quantitative, and mixed method approaches. 4th ed, California: SAGE Publictions, 2014. 


\section{ededucitec}

ISSN: 2446-774X

EDUCAPES. Disponível em: <https://educapes.capes.gov.br/>. Acesso em: 25 mai. 2018.

Google Expeditions. Google For Education. Disponível em: <https://edu.google.com/expeditions/\#about>. Acesso em: 25 maio 2018.

INSTITUTO PENÍNSULA, Desafios reais do cotidiano escolar brasileiro: 22 dilemas vividos por diretores, coordenadores e professores em escolas de todo o Brasil, São Paulo: Moderna, 2018.

LEMOV, D. Aula nota 10: 49 técnicas para ser um professor campeão de audiência, 4 ed., São Paulo: Da Boa Prova, 2011.

MEC. Assessoria de Comunicação. Aulas do mestrado profissional para servidores do MEC têm início. Brasília: MEC, 2017.

MICHAELIS. Dicionários. [online]: MELHORAMENTOS, 2018.

MOREIRA, M. A.; NARDI, R. O mestrado profissional na área de ensino de Ciências e Matemática: Alguns esclarecimentos. Revista Brasileira de Ensino de Ciência e Tecnologia, Ponta Grossa, v. 2, n. 3, p. 1-9, 2009.

Palma Kids. Disponível em: <http://www.palmakids.com/>. Acesso em: 28 maio 2018.

Primo. Primotoys. Disponível em: <https://www.primotoys.com/>. Acesso em: 25 maio 2018.

Star Walk. Disponível em: <http://www.vitotechnology.com/star-walk-kids.html>. Acesso em: 28 maio 2018.

W3C BRASIL. Cartilha Acessibilidade na Web. 2013. Disponível em: $<$ http://www.w3c.br/pub/Materiais/PublicacoesW3C/cartilha-w3cbracessibilidade-web-fasciculo-I.html>. Acesso em: 28 maio 2018.

Submetido em 24/08/2018.

Aceito em 20/02/2019. 